\title{
PORT-ACCESS CORONARY ARTERY BYPASS GRAFTING: A PROPOSED SURGICAL METHOD
}

John H. Stevens, MD

Thomas A. Burdon, MD

William S. Peters, MB, ChB

Lawrence C. Siegel, MD

Mario F. Pompili, MD

Mark A. Vierra, MD

Frederick G. St. Goar, MD

Greg H. Ribakove, MD

R. Scott Mitchell, MD

Bruce A. Reitz, MD

\begin{abstract}
Minimally invasive surgical methods have been developed to provide patients the benefits of open operations with decreased pain and suffering. We have developed a system that allows the performance of cardiopulmonary bypass and myocardial protection with cardioplegic arrest without sternotomy or thoracotomy. In a canine model, we successfully used this system to anastomose the internal thoracic artery to the left anterior descending coronary artery in nine of $\mathbf{1 0}$ animals. The left internal thoracic artery was dissected from the chest wall, and the pericardium was opened with the use of thoracoscopic techniques and single lung ventilation. The heart was arrested with a cold blood cardioplegic solution delivered through the central lumen of a balloon occlusion catheter (Endoaortic Clamp; Heartport, Inc., Redwood City, Calif.) in the ascending aorta, and cardiopulmonary bypass was maintained with femorofemoral bypass. An operating microscope modified to allow introduction of the $3.5 \times$ magnification objective into the chest was positioned through a $10 \mathrm{~mm}$ port over the site of the anastomosis. The anastomosis was performed with modified surgical instruments introduced through additional $5 \mathrm{~mm}$ ports. In the cadaver model $(n=7)$ the internal thoracic artery was harvested and the pericardium opened by means of similar techniques. A precise arteriotomy was made with microvascular thoracoscopic instruments under the modified microscope on four cadavers. In three other cadavers we assessed the exposure provided by a small anterior incision ( 4 to $6 \mathrm{~cm}$ ) over the fourth intercostal space. This anterior port can assist in dissection of the distal internal thoracic artery and provides direct access to the left anterior descending, circumflex, and posterior descending arteries. We have demonstrated the potential feasibility of grafting the internal thoracic artery to coronary arteries with the heart arrested and protected, without a major thoracotomy or sternotomy. (J THORAC CARDIOVASC SURG 1996;111:567-73)
\end{abstract}

$\mathrm{R}^{\mathrm{c}}$ ecent changes in surgical technique and technology have allowed the performance of abdominal and thoracic surgical procedures in ways not imagined even a few years ago. These procedures tend to provide the definitive benefits of major operations, while minimizing the injury of access to the body. In carefully selected patients these methods may re-

From the Departments of Cardiothoracic Surgery, Anesthesia, and Surgery, Stanford University School of Medicine, Stanford, Calif,, and Veterans Affairs Medical Center, Palo Alto, Calif.

Funded in part by a grant from Heartport, Inc., Redwood City, Calif.

Received for publication Nov. 7, 1995; revisions requested Nov. 17, 1995; revisions received Dec. 12, 1995; accepted for publication Dec. 13, 1995.

Address for reprints: Lawrence C. Siegel, MD, Stanford University School of Medicine, 300 Pasteur Dr., Stanford, CA 94305-5117. 12/1/71168 duce the morbidity of surgical intervention. Ligation of patent ductus arteriosus and division of vascular rings ${ }^{1,2}$ are examples of cardiovascular procedures that have been successfully performed by thoracoscopy.

We have recently developed an effective endovascular system for cardiopulmonary bypass (CPB) and myocardial protection. This system uses femoral arterial and venous access for CPB and a transfemoral endoaortic occlusion catheter (Endoaortic Clamp; Heartport, Inc., Redwood City, Calif.). It will provide a platform for performing many different cardiovascular procedures in a potentially less invasive fashion than required by current methods. ${ }^{3}$ This article describes a surgical method for performing "port-access" bypass grafting of the internal thoracic artery (ITA) to coronary arteries. 


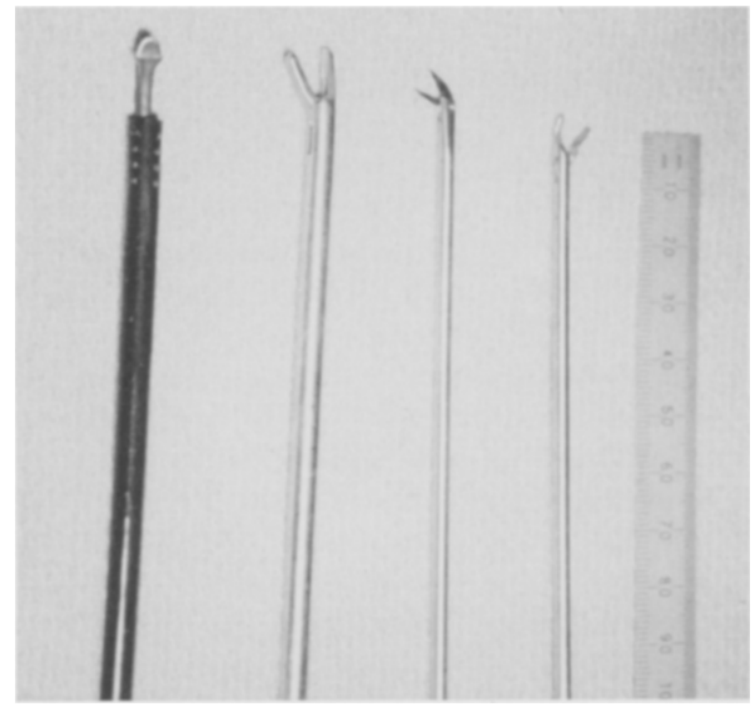

Fig. 1. Instruments used for dissection of the ITA and anastomosis to the LAD. Left to right: Electrocauterysuction-irrigation device, forceps, coronary arteriotomy scissors, microvascular anastomosis suture needle driver.

\section{Method}

Canine studies. Ten mongrel dogs weighing 20 to $30 \mathrm{~kg}$ were used to determine a surgical method for port-access coronary artery bypass grafting (CABG). All animals were given humane care in compliance with the "Principles of Laboratory Animal Care" formulated by the National Society for Medical Research and the "Guide for the Care and Use of Laboratory Animals" prepared by the Institute of Laboratory Animal Resources and published by the National Institutes of Health (NIH Publication No. 86-23, revised 1985).

General anesthesia was initiated and maintained with morphine sulfate and pentobarbital. Single lung ventilation was accomplished with a balloon blocking the left bronchus. Three $10 \mathrm{~mm}$ ports were made in the third, fifth, and seventh intercostal spaces (ICSs) in the midaxillary line. A Distalvu 360 thoracoscope (Welch-Allyn, Skaneatales, N.Y.) was inserted into the lowermost port. The Distalvu camera is designed to allow intracavitary changes in position by way of a fingertip control steering mechanism. A 30-degree angled DeBakey type thoracoscopic forceps (Heartport) was inserted through the middle port. A combination electrocautery, suction, and irrigation device (Heartport), used through the third ICS port, allowed diathermy- assisted dissection with immediate smoke evacuation (Fig. 1). The surgeon controlled the electrocautery and forceps and the assistant guided the endoscope (Fig. 2). Dissection was begun proximally. Once across the pedicle (from lateral to medial), the forceps were passed through and were used to retract the pedicle down to facilitate the dissection. Surgical clips (Liga-clips; Weck, N.C.) were applied to secure side branches of the ITA. Approximately half way through the dissection, the thoracoscope was changed to the third ICS port and the dissecting instruments were moved to the lower two ports to complete the dissection distally. Heparin was administered intravenously (300 U/kg) and two medium/large surgical clips were placed distally across the artery. After the scope had been returned to a lower port, the forceps were inserted through the third ICS port to grasp the fascia of the pedicle just proximal to the distal clips. A thoracoscopic atraumatic temporary occlusion clamp (Heartport) was applied to the ITA pedicle proximally. The artery was divided and exteriorized through the upper port, with care taken to avoid twisting. Any proximal tethering, particularly from the medial aspect, was identified and dissected free to maximize the length of the pedicle. The occlusion clamp was released and flow of the ITA checked. The artery was prepared for manastomosis externally, and a $10 \mathrm{~cm}$ double-armed 7-0 polytetrafluoroethylene suture (Ultex; W. L. Gore \& Associates, Iric., Elkton, Md.) was inserted into the heel.

The Endovascular Cardiopulmonary Bypass System (Heartport) was inserted by way of a femoral artery and vein cutdown (Fig. 3). This system has been fully described (see Schwartz et al., page 556). In brief, femoral arterial and femoral venous access is used for CPB. The venous line is augmented with a centrifugal pump. An Endopulmonary Vent (Heartport), passed via a jugular vein, assists in venting the right side of the heart and pulmonary circulation. The endoaortic balloon occlusion catheter (Endoaortic Clamp) is inflated to occlude the ascending aorta, and cardioplegic solution is delivered by way of a central lumen. It is also routinely used to vent the aortic root by way of the same central lumen. A separate lumen allows continuous transducing of aortic root pressure.

CPB was initiated and the pericardium was incised sharply anterior to the phrenic nerve from the pulmonary artery to the diaphragm. The pedicle was returned inside the chest and positioned on the heart. The coronary arteries were identified by means of the thoracoscope, and port placement for the $10 \mathrm{~mm}$ Stereovision Probe (Heartport) was first determined by passing a needle through the anterior chest wall to locate the most direct line to the proposed anastomosis site. Two to four $5 \mathrm{~mm}$ ports (for microvascular instruments) and a single $10 \mathrm{~mm}$ port (Stereovision Probe) were inserted anteriorly (Fig. 4). The Stereovision Probe was coupled to the operating microscope (OPMI-MDU; Ziess, Thornwood, N.Y.), and a visual check was made.

Under fluoroscopic guidance, the endoaortic balloon was inflated; and high-potassium cold blood $(\mathrm{KCl}, 20$ $\mathrm{mmol} / \mathrm{L}, 4^{\circ} \mathrm{C}$ ) cardioplegic solution was infused into the aortic root.

The Stereovision Probe was positioned in the central 10 $\mathrm{mm}$ anterior port, and the left anterior descending artery (LAD) was identified. A 3 to $4 \mathrm{~mm}$ arteriotomy was made and the ITA was anastomosed to the $\mathrm{LAD}$ with a running continuous suture. A U stitch was placed in the heel of the ITA, with both needles passing from out to in, to facilitate the anastomosis. The needles were then both passed from within the heel of the coronary artery to out, before one arm was sewn up each side toward the toe. On completion of the anastomosis, an instrument tie was performed. The temporary occlusion clamp on the ITA was released and repair sutures were placed if needed. The endoaortic 


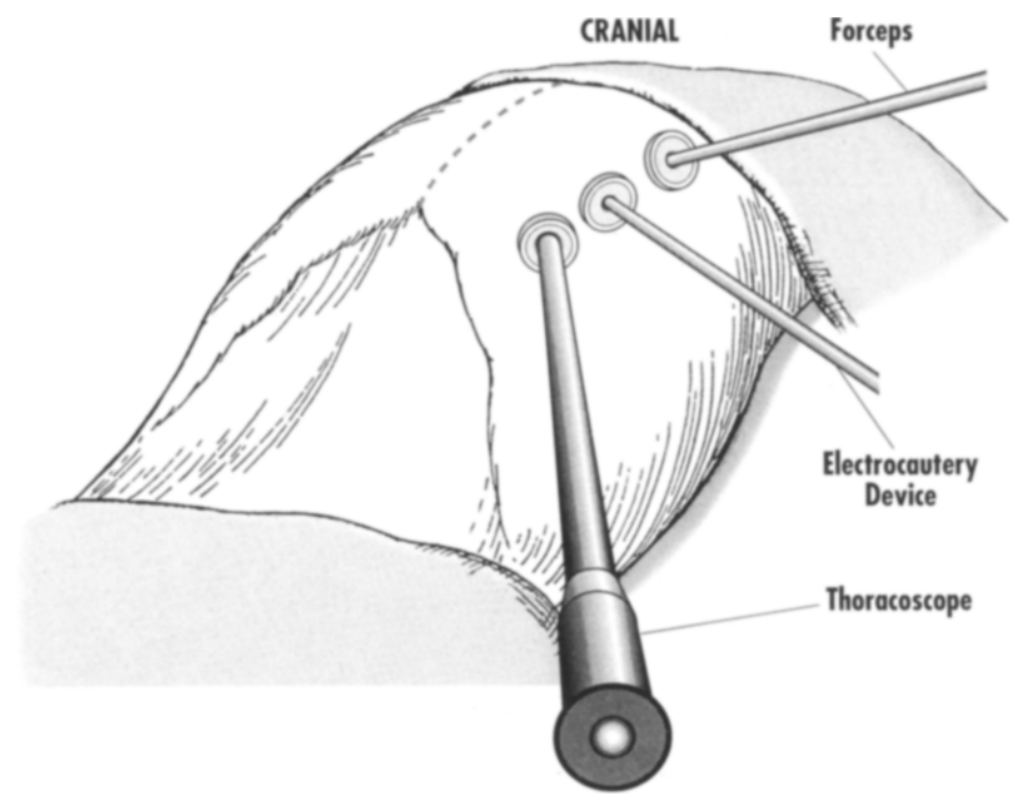

Fig. 2. Thoracoscopic left ITA takedown in the canine model. Ports are made in the third, fifth, and seventh ICSs in the midaxillary line. The pedicle is mobilized proximally first, with the thoracoscope in the lowermost port:

occlusion balloon catheter (Endoaortic Clamp) was deflated and the animal weaned from CPB. The animal was put to death after cardiac output had been measured by the thermodilution technique and an angiogram had been obtained.

Human cadaver study. Human cadavers were obtained from the Stanford University Division of Human Anatomy. Written consent was given by the donor's family for use of the cadaver for medical education and research. Seven cadavers were used to compare and contrast different components of the surgical method used in the canine model.

In four cadavers, three intercostal ports were inserted in the anterior axillary line. The Distalvu 360 thoracoscope was used to visualize the left ITA. The ITA was then fully dissected away from the chest wall from the subclavian vein to the level of the xiphoid process by means of endoscopic dissecting scissors and 30-degree angled DeBakey forceps. A port was made anteriorly to allow countertraction of the pericardium, and the pericardium was opened anterior to the phrenic nerve and rightward to widely expose the heart. The LAD was then located with the thoracoscope, and an anterior port was inserted in the third or fourth ICS in a parasternal location, directly over the target anastomosis site. The Stereovision Probe was inserted, and two to four additional $5 \mathrm{~mm}$ ports were inserted radially around this anterior port to allow the surgeon and assistant bimanual access to the heart for the anastomosis. The LAD was entered in its midportion with a scalpel. If autolysis was not significant, the ITA was anastomosed to the LAD.

In the three other cadavers, an alternative approach to access the heart for the anastomosis was to perform a 4 to
$6 \mathrm{~cm}$ transverse parasternal incision in the fourth ICS. If better exposure was needed, the costal cartilage of an adjacent rib was removed. This space was used to facilitate the thoracoscopic ITA takedown. A soft-tissue retractor (Heartport) was used to maintain a patent port. The distal end of the pedicle was brought out through the anterior port for preparation. The pericardium was opened through this port and extended from the pulmonary artery to the diaphragm by a combination of direct vision and thoracoscopy. Stay sutures were placed to keep the pericardium open and to bring the heart closer to the chest wall. The LAD was identified and an arteriotomy made under direct vision (Fig. 5). Rib spreading was not used.

\section{Results}

Canine studies. In all animals the ITA was well visualized and easily accessed. The distal two thirds of the ITA was typically buried deep to muscle, but we were able to fully mobilize it without injury, and it had good blood flow. The pericardium was easily opened in all animals. The Endovascular Cardiopulmonary Bypass System (Heartport) and endoaortic occlusion catheter (Endoaortic Clamp; Heartport) were applied without difficulty in all cases. The duration of aortic occlusion was $60 \pm 26$ minutes (mean \pm standard deviation) and the duration of CPB was $102 \pm 26$ minutes. Asystole was maintained during aortic occlusion, so that the operative 


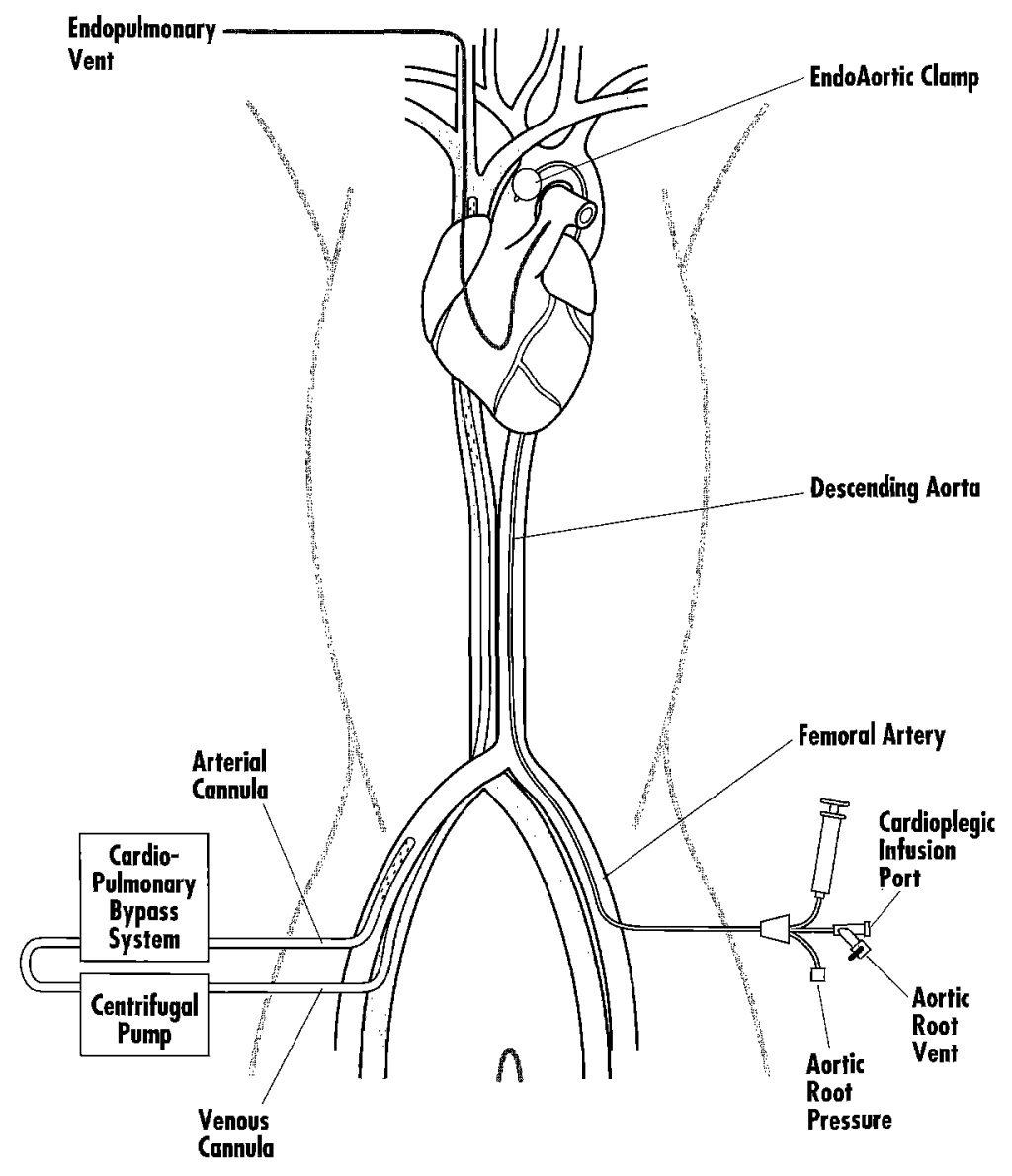

Fig. 3. Endovascular Cardiopulmonary Bypass System. Femorofemoral CPB with a centrifugal pump to augment venous drainage. The balloon occlusion catheter (Endoaortic Clamp) is inflated in the ascending aorta and cardioplegic solution is delivered antegradely through a central lumen. The endopulmonary vent assists decompression of the right side of the heart and pulmonary circulation.

field was still in all animals. All anastomoses were made with the aid of the Stereovision Probe. The total number of ports used ranged from six to eight.

After deflation of the endoaortic balloon (Endoaortic Clamp) and resuscitation to sinus rhythm, all dogs were successfully weaned from $\mathrm{CPB}$ without inotropic support. Cardiac output was $2.5 \pm 0.5$ $\mathrm{L} / \mathrm{min}$ before $\mathrm{CPB}$ and $3.0 \pm 0.6 \mathrm{~L} / \mathrm{min}$ after $\mathrm{CPB}$. By means of angiography, nine of 10 grafts were confirmed to be patent; the pedicles were not twisted and there was brisk runoff with appropriate angulation at the anastomoses. In one animal the surgeon suspected that the anastomosis suture might have passed through the back wall of the LAD. Angiographic studies showed that this anastomosis was not patent, and postmortem examina- tion confirmed the failure. Gross examination of the hearts post mortem showed no evidence of myocardial injury.

Human cadaver studies. Left ITA access and visualization was assessed in all studies. Because of the relatively small anteroposterior diameter in human beings compared with dogs, the distal ITA was more difficult to access. We found that bringing the lower ports into the anterior axillary line aided in the exposure of the distal ITA. The ITA was taken down in all seven studies. Six were taken down, from the first rib to the diaphragm, with the use of three $10 \mathrm{~mm}$ ports and a technique similar to that described for the dog. One ITA takedown was done by unroofing the distal ITA first by removing a small piece of the left fourth costal cartilage. Through this incision the ITA could be transilluminated with the 


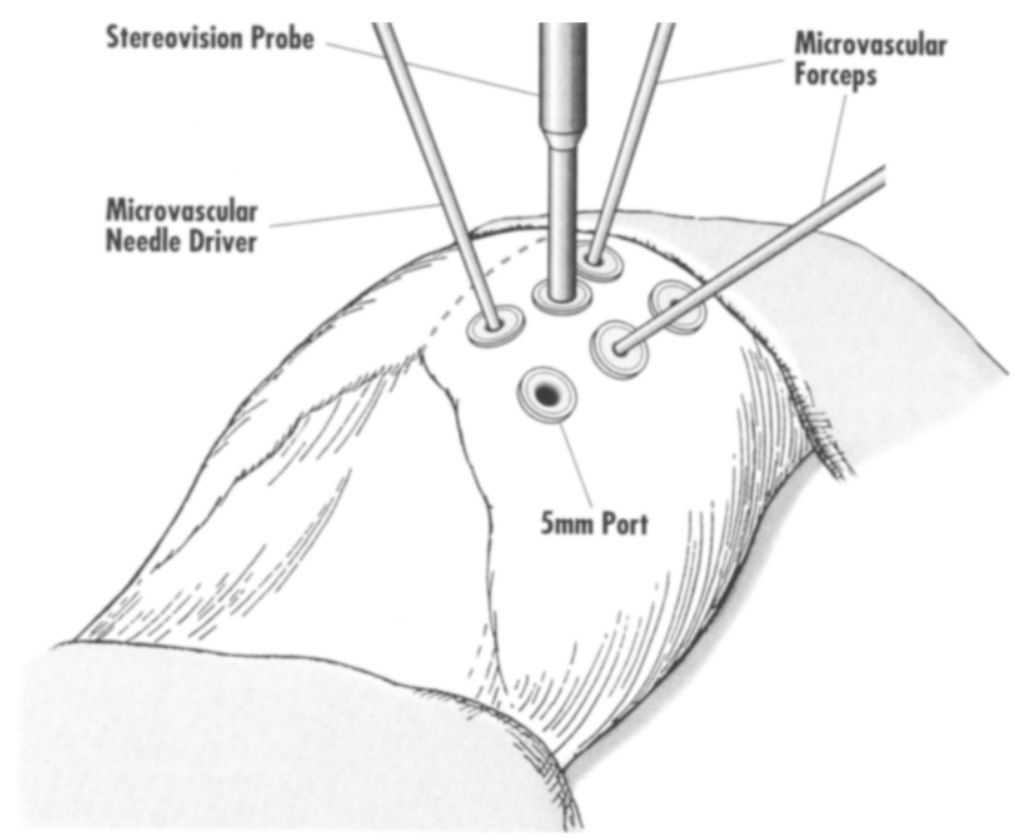

Fig. 4. Thoracoscopic anastomosis in the canine model. The Stereovision Probe is surrounded radially by $5 \mathrm{~mm}$ ports for microvascular anastomosis instruments.

thoracoscope and a pedicle of approximately 4 to 6 $\mathrm{cm}$ could be created. The dissection was completed thoracoscopically.

A pericardiotomy was made in all studies. This was done by complete thoracoscopic access in the first four cadavers and through the anterior incision in the remainder. One of the takedown ports can be extended to make the anastomosis port. The interventricular groove and $\mathrm{LAD}$ were identified and a precise arteriotomy was made in all studies. Visualization of the LAD was considered exceptional with the Stereovision Probe. In the three latter studies, working through an intercostal incision allowed direct access to the LAD and diagonal branches. The heart was able to be retracted to expose the obtuse marginal branches and the posterior descending artery. Removing a section of costal cartilage from the fourth ICS allowed much wider access to the same vessels.

\section{Discussion}

Recent years have seen a tremendous growth in transcatheter management of acquired heart disease, particularly angioplasty of coronary arteries. These techniques have been popular because they are perceived to be reasonably efficacious and to be associated with low morbidity. ${ }^{4,5}$ In many instances, they are not as effective or definitive as open cardiac operations, ${ }^{6}$ but the trade-off of low morbidity and cost makes them attractive.

Recently, techniques of laparoscopy and thoracoscopy have revolutionized many areas of general and thoracic surgery. For example, in 1993, only 4 years after the first laparoscopic cholecystectomy had been performed in the United States, it was estimated that $80 \%$ of cholecystectomies in this country were being performed by laparoscopy. ${ }^{7,8}$ In general thoracic surgery, bleb resection and pleurodesis for spontaneous pneumothorax, ${ }^{9}$ thoracoscopic or laparoscopic Heller myotomy, ${ }^{10}$ and wedge resection of solitary pulmonary lesions ${ }^{11}$ are examples of procedures that have gained widespread acceptance. Some individuals have attempted to use the ITA for CABG using thoracoscopic techniques or minithoracotomy, ${ }^{12,}{ }^{13}$ but not, to our knowledge, in conjunction with a system that would provide myocardial protection comparable with that achieved with open surgical techniques of CPB.

$\mathrm{We}^{14}$ have previously described a method to effectively perform CPB and aortic occlusion with cardioplegic arrest without opening the chest in the canine model. With the endovascular CPB platform it becomes feasible to apply minimally invasive techniques to cardiovascular surgical problems. This 


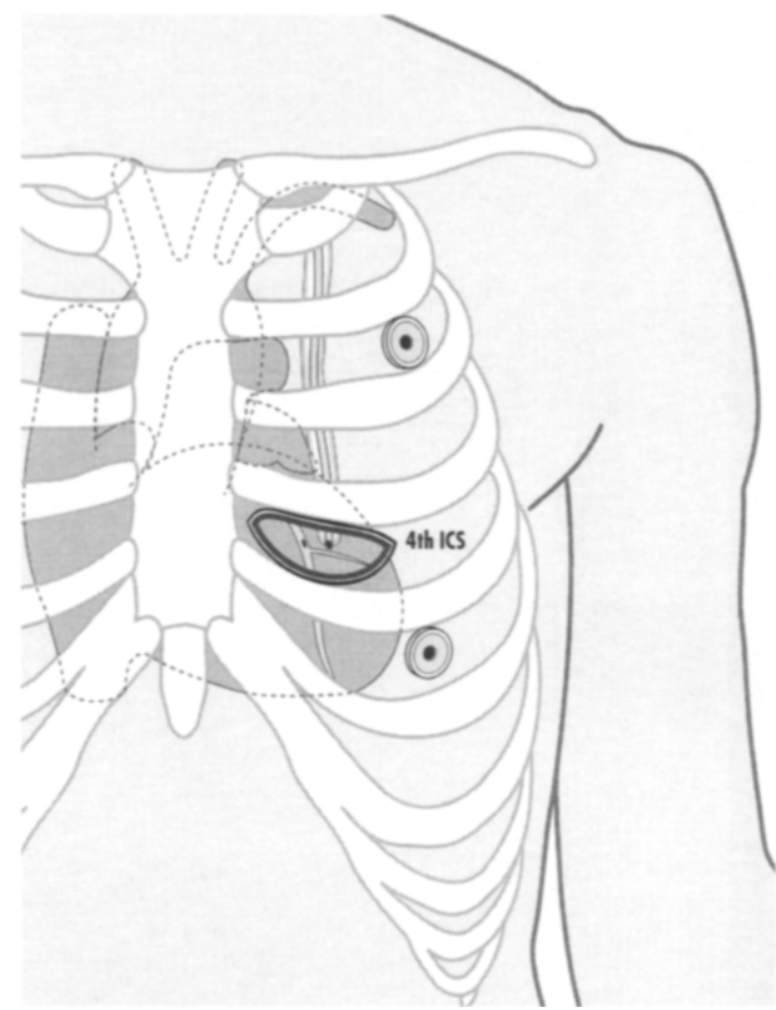

Fig. 5. Anastomosis under direct vision through an anterior port. The fourth ICS port is extended medially 4 to 6 $\mathrm{cm}$ and the soft tissues are retracted to create an anterolateral anastomosis port.

article describes a surgical method in the canine and human cadaver models that allows ITA-LAD anastomoses to be constructed without the need for a median sternotomy or major thoracotomy. The essential components to this approach are, first, an effective and safe means to obtain a quiet and bloodless field so that precise anastomoses can be performed. Second, task-specific instruments for ITA dissection and coronary anastomoses are necessary. Finally, high-quality stereoscopic visualization is essential. Our surgical methods as described allow either for an operating microscope modified with a $10 \mathrm{~mm}$ Stereovision Probe or for direct visualization with surgical loupes. Using these techniques, as well as techniques borrowed from laparoscopy, thoracoscopy, and microsurgery, we have proposed a method for performing closed chest ITA-LAD anastomosis. We believe that with these techniques it may be possible to duplicate the advantages of traditional open-chest $\mathrm{CABG}$ opera- tions, while avoiding much of the morbidity associated with sternotomy.

The obvious shortcomings to this description of a surgical method for minimally invasive $\mathrm{CABG}$ are twofold. First, a canine model is quite different from the human being. The most significant difference between the dog and human being is the shape of the chest. The flatter, broader chest in human beings makes the left ITA dissection more difficult, particularly distally. In addition, the proximity of the anterior part of the heart to the chest wall somewhat limits the ability to manipulate instruments for creating an anastomosis with a totally closed chest system. The alternative anterior incision provides broader visualization and access, yet may cause less morbidity and discomfort than a median sternotomy. ${ }^{15}$ Multiple grafts are also technically possible with this latter approach; the arrested and protected heart allows for more time and better exposure of the left lateral and posterior aspects of the heart. This incision has allowed CABG without CPB in selected patients (V. Subramenium, personal communication). Performing ITA-coronary anastomoses on the beating heart with limited access restricts the indications of this operation to severe LAD disease. ${ }^{12,13}$

A second problem with this study is that even in the cadaver model, the postmortem changes allow at best only an approximation of the application of these methods to patients. We do believe that this description may set forth a foundation for the further development of minimally invasive $\mathrm{CABG}$ in a wide range of patients.

A clinical trial is presently underway to evaluate the safety and effectiveness of a port-access method as compared with standard $\mathrm{CABG}$ through a median sternotomy. The results of this trial should help define the appropriate treatment paradigm for selected patients with coronary artery disease.

\section{REFERENCES}

1. Laborde F, Noirhomme P, Karam J, et al. A new videoassisted thoracoscopic surgical technique for interruption of patent ductus arteriosus in infants and children. J THORAC Cardiovasc Surg 1993;105:278-80.

2. Burke RP, Rosenfeld HM, Wernovsky G, Jonas RA. Videoassisted thoracoscopic vascular ring division in infants and children. J Am Coll Cardiol 1995;25:943-7.

3. Peters WS. Minimally invasive cardiac surgery by cardioscopy. Australas J Thorac CardiovasC Surg 1993;2:152-4.

4. Hamm CW, Reimers $\mathbf{J}$, Ischinger $\mathrm{T}$, et al. A randomized study of coronary angioplasty compared with bypass surgery in patients with symptomatic multivessel coronary disease. 
German Angioplasty Bypass Surgery Investigation (GABI). N Engl J 1994;331:1037-43.

5. King SB III, Lembo NJ, Weintraub WS, et al. A randomized trial comparing coronary angioplasty with coronary bypass surgery: Emory Angioplasty versus Surgery Trial (EAST). N Engl J Med 1994;331:1044-50.

6. Boylan MJ, Lytle BW, Loop FD, et al. Surgical treatment of isolated left anterior descending coronary stenosis: comparison of left internal mammary artery and venous autograft at 18 to 20 years of follow-up. J THORAC CARDIOvasC SURG 1994;107:657-62.

7. Begos DG, Modlin IM. Laparoscopic cholecystectomy: from gimmick to gold standard. J Clin Gastroenterol 1994;19:32530.

8. Development panel on gallstones and laparoscopic cholecystectomy: NIH Consensus. JAMA 1993;269:1018-24.

9. Waller DA, Forty J, Morritt GN. Video-assisted thoracoscopic surgery versus thoracotomy for spontaneous pneumothorax. Ann Thorac Surg 1994;58:372-6.
10. Pellegrini CA, Leichter R, Patti M, Somberg K, Ostroff JW, Way L. Thoracoscopic esophageal myotomy in the treatment of achalasia. Ann Thorac Surg 1993;56:680-2.

11. Mack MJ, Hazelrigg SR, Landreneau RJ, Acuff TE. Thoracoscopy for the diagnosis of the indeterminate solitary pulmonary nodule. Ann Thorac Surg 1993;56:825-30.

12. Benetti FJ, Ballester C. Use of thoracoscopy and a minimal thoracotomy, in mammary-coronary bypass to left anterior descending artery, without extracorporeal circulation: experience in 2 cases. J Cardiovasc Surg 1995;36:159-61.

13. Robinson MC, Gross DR, Zemen W, Stedje-Larsen E. Minimally invasive coronary artery bypass grafting: a new method using an anterior mediastinotomy. J Card Surg 1995;10:529-36.

14. Stevens JH, Siegel LC, Mitchell RS, et al. Closed-chest coronary artery bypass with cardioplegic arrest in the dog. Circulation 1994;90(4 Pt 2):251.

15. Vallieres E, Page A, Verdant A. Ambulatory mediastinoscopy and anterior mediastinotomy. Ann Thorac Surg 1991;52:1122-6.

\section{ON THE MOVE?}

Don't miss a single issue of the journal! To ensure prompt service when you change your address, please photocopy and complete the form below.

Please send your change of address notification at least six weeks before your move to ensure continued service. We regret we cannot guarantee replacement of issues missed due to late notification.

\section{JOURNAL TITLE:}

Fill in the title of the journal here.

\section{OLD ADDRESS:}

Affix the address label from a recent issue of the journal here.

\section{NEW ADDRESS:}

Clearly print your new address here.

Name

Address

City/State/ZIP
COPY AND MAIL THIS FORM TO:

Journal Subscription Services

Mosby-Year Book, Inc.

11830 Westline Industrial Dr.

St. Louis, MO 63146-3318
OR FAX TO:

314-432-1158

N/Mosby
OR PHONE:

1-800-453-4351

Outside the U.S., call

314-453-4351 